\title{
Significance of Theoretical Decomposition Enthalpies for Predicting Thermal Hazards
}

\author{
Didier Mathieu \\ CEA, DAM, Le Ripault, 37260 Monts, France \\ Correspondence should be addressed to Didier Mathieu; didier.mathieu@cea.fr
}

Received 24 March 2015; Accepted 21 May 2015

Academic Editor: Robert Zaleśny

Copyright ( 2015 Didier Mathieu. This is an open access article distributed under the Creative Commons Attribution License, which permits unrestricted use, distribution, and reproduction in any medium, provided the original work is properly cited.

\begin{abstract}
Much effort is currently put into the development of models for predicting decomposition enthalpies measured using differential scanning calorimetry (DSC). As an alternative to the purely empirical schemes reported so far, this work relies on theoretical values obtained on the basis of simple assumptions. For nitroaromatic compounds (NACs) studied in sealed sample cells, our approach proves clearly superior to previous ones. In contrast, it correlates poorly with data measured in pin-hole sample cells. Progress might be obtained through a combination of the present approach with the usual Quantitative Structure-Property Relationships (QSPR) methodologies. This work emphasizes the significance of the theoretical decomposition enthalpy as a fundamental descriptor for the prediction of DSC values. In fact, the theoretical value provides a valuable criterion to characterize thermal hazards, as a complement to experimental decomposition temperatures.
\end{abstract}

\section{Introduction}

In view of the increasingly large number of compounds to be considered in process design or in the context of recent regulatory frameworks, there is currently much interest in the numerical estimation of thermal hazards prior to any experiment [1]. Such hazards are classified and predicted on the basis of several criteria [2], including exothermic onset temperatures $\left(T_{0}\right)$ and decomposition enthalpies derived from differential scanning calorimetry (DSC) experiments [3-5]. The latter property, hereafter denoted by $\Delta_{d} H$ (DSC) and defined as positive, is a preliminary to any assessment of thermal risks. It is a relatively simple property which may be loosely defined as the opposite of the enthalpy change associated with the decomposition of the compound studied into products. Assuming that this process yields the most stable products consistent with the stoichiometry of the system, we obtain an ideal value $\Delta_{d} H^{0}$ that can be identified with the energy content of the material.

The difference between $\Delta_{d} H^{0}$ and $\Delta_{d} H$ (DSC) is determined by highly complex processes, including multiple reaction pathways, secondary reactions possibly involving the surroundings of the sample, and heat and mass transport processes within the cell. A first-principles approach to this difference is therefore out of reach, considering the computational cost inherent to ab initio molecular dynamics and the fact that reactive potentials, which could provide a more efficient alternative, are still in their infancy [6]. Therefore, some empiricism is unavoidable when it comes to predicting DSC data, and Quantitative Structure-Property Relationships (QSPR) methodologies appear as a natural approach to estimate $\Delta_{d} H^{0}-\Delta_{d} H$ (DSC) in this context.

Although they do rely on such techniques, presently published models apply them directly to the evaluation of $\Delta_{d} H$ (DSC) in terms of standard descriptors [7-15]. As a result, they do not take advantage of the availability of simple rules to estimate decomposition products and corresponding $\Delta_{d} H^{0}$ values [16]. While QSPR approaches prove extremely useful in the lack of quantitative theories, especially for complex properties in the fields of pharmaceutical chemistry, toxicology, or risk assessment, they are some reasons to believe that their direct application to $\Delta_{d} H$ (DSC) is unlikely to provide the most reliable predictive tools.

First, QSPR descriptors are calculated on the unreacted compound, while $\Delta_{d} H$ (DSC) also depends on the decomposition products and possibly on some features of the reaction 
pathways. Secondly, $\Delta_{d} H$ (DSC) obviously depends on the formation enthalpy $\Delta_{f} H^{0}$ of the compound studied. Since a reliable evaluation of this property for arbitrary compounds requires quantum chemical computations, QSPR models are unlikely to provide accurate $\Delta_{d} H$ (DSC) values for a wide range of compounds.

Finally, an even more significant obstacle to the application of QSPR techniques to estimate $\Delta_{d} H$ (DSC) is the lack of consistent data. The routine application of DSC to thermal hazard evaluation yields decomposition enthalpies with experimental uncertainties around 10\% [4]. However, a more significant variability is observed in practice due to the details of the experimental setup, including the temperature scanning rate, the materials of the device, or the kind of sample cell. For instance, on going from a sealed [3] to a pinhole [4] sample cell, the measured decomposition enthalpies decrease from 345 to $123 \mathrm{~kJ} / \mathrm{mol}$ for 2-nitrophenol, from 284 to $130 \mathrm{~kJ} / \mathrm{mol}$ for 1-methyl-3-nitrobenzene, and from 339 to $161 \mathrm{~kJ} / \mathrm{mol}$ for nitrobenzene. The relative scarsity of homogeneous $\Delta_{d} H$ (DSC) data sets makes the parametrization and validation of QSPR models very difficult and prevents the introduction of many empirical parameters that might be necessary for reliable predictions of $\Delta_{d} H$ (DSC) along such lines.

In fact, a growing body of work demonstrates the interest of more physically grounded and/or less empirical strategies to estimate the physical properties of organic substances [17-25], including complex properties characterizing reactive hazards such as flash point temperatures [23], flammability limit temperatures [24], or mechanical sensitivities of explosives [25]. Taking advantage of theoretical $\Delta_{d} H^{0}$ values, the present work investigates a semiempirical approach to the evaluation of $\Delta_{d} H$ (DSC) for nitroaromatic compounds (NACs), as an alternative to the straightforward application of QSPR techniques. More specifically, two predictive equations are put forward. The first one simply assumes that $\Delta_{d} H$ (DSC) can be reasonably approximated as a constant fraction $f$ of the energy content $\Delta_{d} H^{0}$, while the second one attempts to describe how this fraction depends on the reactivity of the compound.

\section{Theory}

In a first step, the present approach involves only simple thermodynamic considerations. In a second step, density functional theory (DFT) concepts are invoked in an attempt to introduce kinetic factors. Because $\Delta_{d} H$ (DSC) primarily reflects the heat released as the sample decomposes, the energy content $\Delta_{d} H^{0}$ of the substance should provide a major contribution to measured DSC values. The difference between both quantities may be further decomposed into three correction terms accounting, respectively:

(1) for the fact that the equilibrium products relevant to the thermodynamic conditions in the sample cell may differ from the most stable ones in standard conditions $\left(\delta_{\text {eq }}\right)$;

(2) for the fact that kinetics may affect the actual composition of the products $\left(\delta_{\mathrm{kin}}\right)$;
(3) for energy losses associated with heat and matter transport processes $\left(\delta_{\mathrm{DSC}}\right)$ :

$$
\Delta_{d} H^{0}-\Delta_{d} H(\mathrm{DSC})=\delta_{\mathrm{eq}}+\delta_{\mathrm{kin}}+\delta_{\mathrm{DSC}} .
$$

While $\Delta_{d} H^{0}$ may be estimated on the basis of standard approximations, there is no simple way to calculate the three terms on the r.h.s. of this equation. Therefore, we follow a simpler approach, assuming in a first step that each correction represents a given fraction of $\Delta_{d} H^{0}$. As a result,

$$
\Delta_{d} H(\mathrm{DSC})=f \Delta_{d} H^{0},
$$

where $f$ is a constant that does not depend on the compound under consideration. An advantage of this approach is that it automatically ensures the condition $\Delta_{d} H$ (DSC) $<\Delta_{d} H^{0}$.

Going one step further, we are faced with the challenge to estimate how $f$ depends on the compound under study. Among many factors that are likely to affect the value of this ratio, reactivity might be the one whose role is easiest to describe at least qualitatively. Indeed, a complete decomposition requires that the products be slowly cooled down to the ambient temperature. Such complete decomposition is especially unlikely for materials that remain chemically unaffected up to high temperatures. As temperature increases, the system can explore a larger fraction of the potential energy surface (PES) and is thus more likely to get stuck in metastable configurations during cooling. Therefore, the amount of energy eventually released in decomposition processes depends on the energy barriers $E^{\neq}$.

In the lack of detailed knowledge of reaction pathways, we have to be satisfied with the features of the initial and final states to evaluate the role of $E^{\neq}$. On the products side, the Bell-Evans-Polanyi principle [26] suggests that $E^{\neq}$is likely to get lower as $\Delta_{d} H^{0}$ increases. This principle is frequently invoked to correlate reaction properties with a difference between reactants and products, especially in the field of high energy compounds [27]. Nevertheless, it is not sufficient to quantitatively estimate energy barriers, as reflected by the lack of correlation between decomposition enthalpies and decomposition temperatures [4].

Some improvement might be obtained by taking advantage of reactant features. In particular, bond dissociation energies (BDEs) appear to be ideal descriptors in view of evaluating $E^{\neq}[28]$. However, they may be tedious to compute, requiring the optimization of complex open-shell species if large molecules are considered. An attractive alternative is provided by reactivity descriptors at the basis of conceptual DFT [29], namely, electronegativity $(\chi)$ and chemical hardness $(\eta)$. In fact, an early attempt to predict DSC measurements from empirical relationships already assumed that those quantities play a primary role [8].

In the present work, the reactivity descriptors are introduced with the help of dimensionality considerations. The simplest dimensionless quantity that may be defined on the basis of $\chi$ and $\eta$ is their ratio $\chi / \eta$, where $\eta$ is implicitly multiplied by the electron charge $e=1$ in atomic units. 
Therefore, assuming that $f$ depends on $\chi$ and $\eta$, it should then be expandable as a power series:

$$
f=f_{0}+a\left(\frac{\chi}{\eta}\right)+b\left(\frac{\chi}{\eta}\right)^{2}+\cdots .
$$

Since the principle of maximum hardness implies that $\eta$ is a measure of the stability of the system [29], $f$ is expected to decrease as $\eta$ increases, with the limit of large $\eta$ values corresponding to an increasing resistance of the molecule to changes in its electronic structure, hence to energy release. This implies that $a>0$ since the linear term is predominant for large values of $\eta$. On the other hand, setting $f_{0}=0$ is required if we want the energy released to decrease to zero in the hypothetical limit of an infinitely stable system with $\eta \rightarrow \infty$. This suggests a possibly improved alternative to (2):

$$
\Delta_{d} H(\mathrm{DSC})=a\left(\frac{\chi}{\eta}\right) \Delta_{d} H^{0} .
$$

In what follows, both (2) and (4) are considered to predict $\Delta_{d} H$ (DSC). Given many factors that may affect measured values, depending on the detailed experimental setup, associated with the approximate character of conceptual DFT inherent to the fact that it attempts to describe reactivity in term of features of the unreacted compound, it is not a priori obvious to decide which equation will prove most reliable.

An attractive feature of present models is the fact that they depend on a single input variable $x$, where $x=\Delta_{d} H^{0}$ if a constant fraction $f$ of the energy content is assumed to contribute to measured DSC data as in (2), or $x=$ $(\chi / \eta) \Delta_{d} H^{0}$ if this fraction is assumed to depend linearly on $\chi / \eta$ as in (4). As a consequence, their performances may be straightforwardly estimated, for instance, graphically from the plot of $\Delta_{d} H$ (DSC) versus $x$, or by considering the determination coefficient $R^{2}$ between both quantities. This is especially gratifying in view of the scarcity of homogeneous data, which make it awkward to define statistically significant external test sets for multiparameters models.

\section{Computational Details}

In this work, the decomposition products are obtained on the basis of a simple generalization to halogenated compounds of the well-known $\mathrm{H}_{2} \mathrm{O}-\mathrm{CO}_{2}$ arbitrary initially introduced to estimate the heats of detonation of $\mathrm{C}-\mathrm{H}-\mathrm{N}-\mathrm{O}$ explosives [30]. More specifically, decomposition products are obtained according to the following priority order: $\mathrm{HF}>\mathrm{CF}_{4}>\mathrm{H}_{2} \mathrm{O}$ $>\mathrm{CO}_{2}>\mathrm{CCl}_{4}>\mathrm{CO}>\mathrm{HCl}$. If necessary, the remaining elements are converted into graphite, $\mathrm{H}_{2}, \mathrm{~N}_{2}, \mathrm{~S}_{8}, \mathrm{O}_{2}, \mathrm{~F}_{2}$, and $\mathrm{Cl}_{2}$. In practice, the last three products are never obtained for the present data set because of the relative scarcity of $\mathrm{O}, \mathrm{F}$, and $\mathrm{Cl}$ atoms in present NACs.

The energy content $\Delta_{d} H^{0}$ is then obtained as the difference between the formation enthalpy $\Delta_{f} H^{0}$ of the substance under study and corresponding values $\Delta_{f} H^{0}(k)$ for the decomposition products $k$. The latter are taken from the NIST Webbook database [31]. Because experimental values of $\Delta_{f} H^{0}$ are missing for most NACs under study, theoretical values computed using the semiempirical RM1 Hamiltonian are used. This method is chosen for its relatively good performance and computational efficiency [32].

For (4), values of $\chi$ and $\eta$ are obtained within the finite difference approach by simple difference and as an average of the ionization potential (IP) and electronegativity (EA):

$$
\begin{aligned}
& \chi=\frac{\mathrm{IP}+\mathrm{EA}}{2}, \\
& \eta=\mathrm{IP}-\mathrm{EA} .
\end{aligned}
$$

IP and EA are derived from the energies $\epsilon_{\mathrm{HOMO}}$ and $\epsilon_{\mathrm{LUMO}}$ of the highest occupied and lowest unoccupied molecular orbitals, obtained simply as a by-product of the RM1 computations. More specifically, IP $=-\epsilon_{\mathrm{HOMO}}$ and $\mathrm{EA}=-\epsilon_{\mathrm{LUMO}}$ [29]. All RM1 calculations are carried out using the MOPAC7 program [33].

Typical errors associated with RM1 formation enthalpies are about $20 \mathrm{~kJ} / \mathrm{mol}$ [32] while experimental uncertainties may cause much larger errors $(>100 \mathrm{~kJ} / \mathrm{mol})$. Therefore, no significant improvement is expected from the use of more accurate procedures. Larger theoretical uncertainties might possibly arise from the use of the RM1 orbitals to obtain the reactivity descriptors. Therefore, we have also investigated in unpublished work the use of enthalpies and orbital energies derived from $\mathrm{PBE} 0 / 6-31+\mathrm{G}(\mathrm{d}, \mathrm{p}) / / \mathrm{AM} 1$ calculations combined with simple atom equivalent schemes [34]. It turned out that this higher theoretical level does not provide any significant improvement with respect to RM1-based procedures. Because RM1 calculations are much easier to carry out routinely in an industrial context, only the results obtained on this basis are presented in the sequel.

Similarly, we investigated the effect of taking into account the contribution of intermolecular interactions to $\Delta_{d} H^{0}$ despite the introduction of sublimation enthalpies calculated on the basis of simple models $[35,36]$. Again, this does not significantly affect the results, as expected from the relatively small magnitude of sublimation enthalpies. Moreover, this approach is not rigorous as the compounds studied typically melt before undergoing a decomposition. Therefore, it is better to ignore the intermolecular contribution to $\Delta_{d} H^{0}$ until a more satisfactory approach is developed.

On the other hand, as an alternative to the abovementioned decomposition rules assuming a complete oxidation of hydrogen and carbon into $\mathrm{CO}_{2}$ whenever possible, the use of the Kistiakowsky-Wilson rules (applying the modified version for compounds with $\mathrm{CO}_{2}$ oxygen balance $<-40 \%$ ) was also considered [16]. These rules favor the formation of $\mathrm{CO}$ over $\mathrm{CO}_{2}$. This change has a more significant impact on the individual results than going from RM1 to PBE0 electronic structures or introducing sublimation enthalpies. Nevertheless, the overall performance of the models is not significantly affected. Therefore, only the results based on the generalized $\mathrm{H}_{2} \mathrm{O}-\mathrm{CO}_{2}$ arbitrary are presented in this work. In the lack of significantly better rules, this is the most attractive option, implying that the effect of any incomplete oxidation is implicitly taken into account through the values of the empirical parameters. 


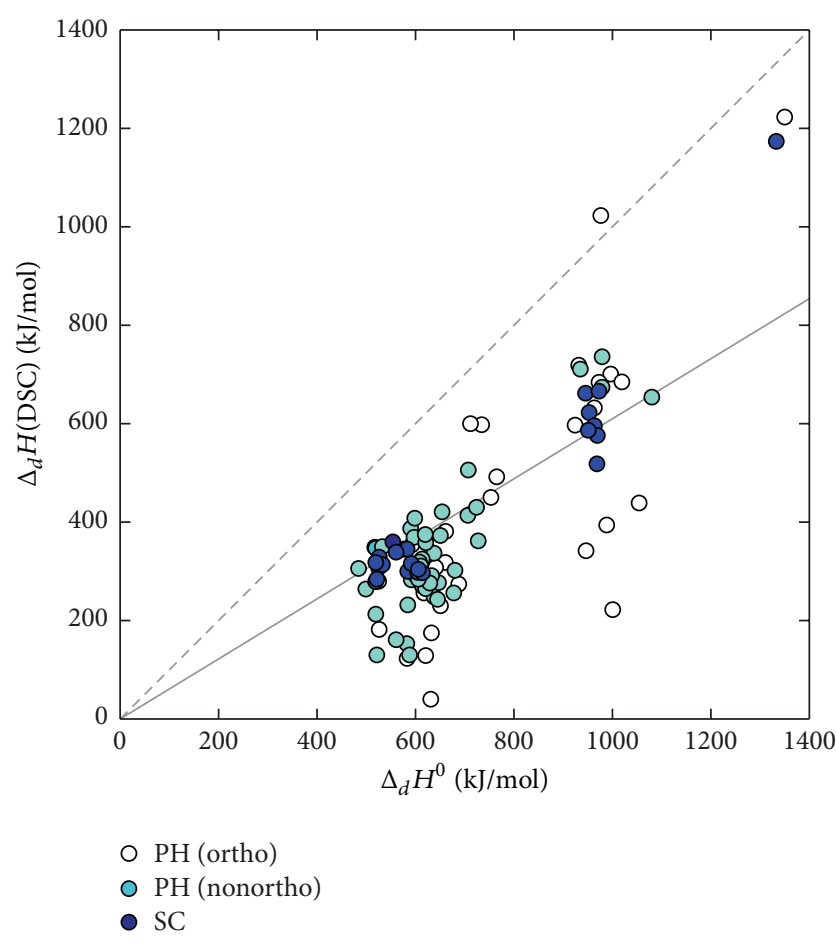

FIGURE 1: Experimental decomposition enthalpies $\Delta_{d} H$ (DSC) plotted against theoretical maximal values $\Delta_{d} H^{0}$. For clarity, the regression line $y=0.61 x$ and the line $y=x$ are indicated as well.

\section{Data Sets}

Recent studies aimed at predicting decomposition enthalpies of NACs as measured by DSC focus on two data sets. The first one is a set of 22 enthalpies measured using a standard aluminium sealed sample cell (SC data) [3]. The second one is a set of 77 enthalpies measured in aluminium sample cells with a pin-hole on the lid ( $\mathrm{PH}$ data) [4]. Technical details regarding these sample cells may be found in references cited in these earlier publications [3, 4]. Most modeling studies focus on the SC data set [8-12]. Only very recent ones take advantage of the $\mathrm{PH}$ data set $[13,15]$. Both SC and $\mathrm{PH}$ data sets are considered in the present work. They provide 99 experimental decomposition enthalpies for 84 compounds, because some molecules have been studied using both kinds of sample cells and are thus present in the two sets.

\section{Results}

5.1. Observed versus Theoretical Enthalpies. Figure 1 compares experimental $\Delta_{d} H$ (DSC) data with theoretical values $\Delta_{d} H^{0}$. Because the kind of sample cell is likely to influence the measured enthalpies, SC and $\mathrm{PH}$ data are shown using different colors. Furthermore, for the analysis of the $77 \mathrm{PH}$ values at hand, the 35 compounds with substituents in ortho position with respect to nitro groups (ortho compounds) are treated separately, assuming that the associated $\Delta_{d} H$ (DSC) values might prove more difficult to rationalize than corresponding data for the remaining 42 compounds deprived of

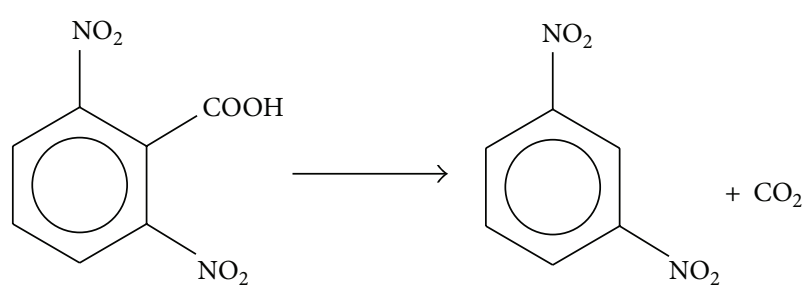

SCHEME 1

ortho substituents (nonortho compounds). This assumption is motivated by the possible role of interactions between nitro groups and corresponding ortho substituents. This partition of the data set between nonortho/ortho compounds was introduced in a previous study [13].

Because $\Delta_{d} H^{0}$ characterizes the total energy content of the compound studied, it should be larger than $\Delta_{d} H$ (DSC). Figure 1 shows that this is actually the case for all compounds except for 2-chloro-3,5-dinitrobenzoic acid which lies slightly above the line $y=x$. This anomaly may be attributed to uncertainties regarding the experimental $\Delta_{d} H$ (DSC) value from the $\mathrm{PH}$ data set and/or the computational procedure to derive $\Delta_{d} H^{0}$.

A correlation between theory and experiment is observed in Figure 1, as reflected by the corresponding determination coefficient $R^{2}=0.68$ which is significantly nonzero. However, the distinction of the different subsets clearly shows that the correlation is especially good for SC data, with $R^{2}=0.90$.

For $\mathrm{PH}$ data, the correlation is less good, although it remains significant with $R^{2}=0.62$. The distinction between nonortho and ortho compounds is especially fruitful here. Indeed, it is clear from Figure 1 that the latter (shown as white symbols) fit especially poorly into the correlation. On the other hand, among the 22 SC values, 10 are for ortho compounds. Therefore, it appears that reactions between nitro groups and neighboring substituents are likely to affect significantly $\Delta_{d} H$ (DSC) for measurements in pin-hole sample cells, while no such effect is noted for experiments in sealed cells.

In fact, $\mathrm{PH}$ values appear to be prone to significant uncertainties, as already mentioned for 2-chloro-3,5dinitrobenzoic acid. As illustrated by the data reported in the introduction to this paper to illustrate the role of the sample cell, PH enthalpies tend to be smaller than SC values, which might be explained by more significant energy losses through evaporation or sublimation of the sample in the former case.

The outliers noted in Figure 1 may be rationalized on a case-by-case basis. For instance, the most significant deviation between $\Delta_{d} H$ (DSC) and $\Delta_{d} H^{0}$ is observed for 2,6dinitrobenzoic acid, for which pin-hole measurements yield $\Delta_{d} H(\mathrm{DSC})=222 \mathrm{~kJ} / \mathrm{mol}$, to be compared with $\Delta_{d} H^{0}=$ $1001 \mathrm{~kJ} / \mathrm{mol}$. By analogy with the synthesis of trinitrobenzene from 2,4,6-dinitrobenzoic acid, this compound is expected to undergo a decarboxylation upon heating, leading to the formation of 1,3-dinitrobenzene and the release of carbon dioxide (see Scheme 1).

Thermochemical data from the NIST Webbook [31] as well as RM1 calculations indicate that this reaction is 
almost athermic. It is thus likely to get unnoticed in DSC experiments. The first reactive exotherm is then associated with the decomposition of 1,3-dinitrobenzene, for which a significantly higher value $\Delta_{d} H($ DSC) $=587 \mathrm{~kJ} / \mathrm{mol}$ was measured in a sealed sample cell. Such artefacts/complexities are clearly not properly taken into account by current predictive methods. Until new models taking advantage of the knowledge of organic chemists are developed, it will probably not be possible to reliably predict measured $\Delta_{d} H$ (DSC) data for arbitrary organic compounds.

It was noted previously that $\Delta_{d} H$ (DSC) data measured in sealed sample cells for NACs primarily depend on the number $n\left(\mathrm{NO}_{2}\right)$ of nitro groups on the molecule [8]. Figure 1 shows that this is systematically the case for $\Delta_{d} H^{0}$ as well, hence the three clusters clearly observed on this plot, corresponding to $n\left(\mathrm{NO}_{2}\right)$ values ranging from 1 to 3 . However, this prominent role of $n\left(\mathrm{NO}_{2}\right)$ is blurred when considering $\mathrm{PH}$ data, as clear from the vertical scattering of the corresponding points, which is especially significant for ortho compounds. Therefore, rationalizing present DSC data should be easiest for SC data and most difficult for ortho compounds characterized in pin-hole sample cells.

For SC data, Figure 1 suggests that rough $\Delta_{d} H$ (DSC) estimates can be obtained by defining three standard values associated, respectively, with mononitro, dinitro, and trinitro compounds. However, the success of such an approach would clearly be highly dependent on the other substituents in the molecule. For instance, it would yield spurious predictions for compounds with other energetic moieties, such as $\mathrm{N}=\mathrm{O},-\mathrm{O}-\mathrm{N}=\mathrm{O},-\mathrm{NF}_{2}$, or $\mathrm{N}_{3}$ groups. Therefore, assuming that $\Delta_{d} H$ (DSC) $\propto \Delta_{d} H^{0}$ as done in (2) is a more attractive approach. However, it is clear from the regression line in Figure 1 that this straightforward scaling relationship does not satisfactorily account for the high decomposition enthalpy obtained for the only trinitrocompound in the SC data set, namely, picric acid. For this molecule, the ratio $\Delta_{d} H$ (DSC) $/ \Delta_{d} H^{0}$ is close to 0.9 , while it is close to 0.6 for mononitro and trinitro compounds.

5.2. Accounting for the Role of Reactivity. This observation actually motivated the considerations leading to (4) in Section 2. Indeed, the relatively large fraction of the energy content released by picric acid may be correlated with the fact it decomposes at a relatively low temperature of $220^{\circ} \mathrm{C}$, the lowest among all compounds studied in sealed cells [3]. As discussed in Section 2, low temperatures favor decomposition according to the lowest energy pathways and therefore more significant releases of energy. Prior to investigating the performances of (4), it is thus interesting to plot $f$ according to $\chi / \eta$ as done in Figure 2. Of course, the relatively poor correlations observed on this figure arise because it focuses on the challenging part of $\Delta_{d} H$ (DSC).

As anticipated, picric acid exhibits specially high value of this ratio. Therefore, (4) should better account for the decomposition enthalpy of this compound compared to (2). The correlation coefficients reported in Figure 2 are systematically positive, hence supporting the considerations leading to (4). To some extent, the higher value obtained for

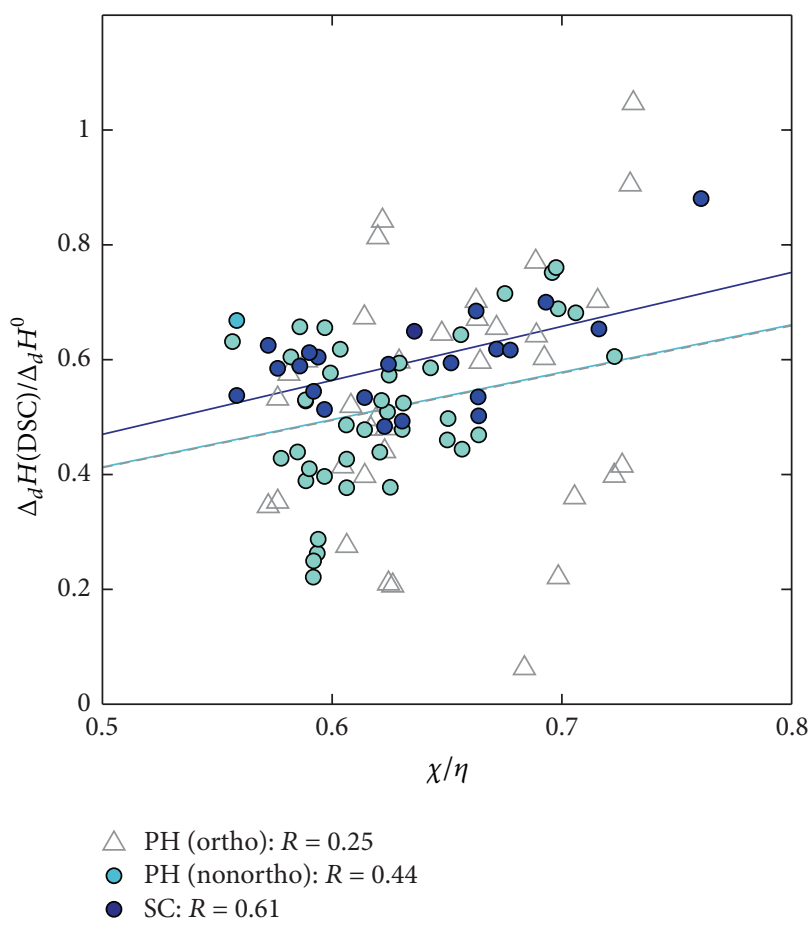

FIgURE 2: Correlations between $f$ and $\chi / \eta$. The regression line for SC data is shown in dark blue. Note that, for PH data, the corresponding lines for nonortho and ortho compounds are the sames (light blue/grey lines). The rightmost symbol stands for the $\mathrm{SC}$ value for picric acid.

SC data may be attributed to the success of the approach to account for the fact that specially high fraction of $\Delta_{d} H^{0}$ contributes to the decomposition enthalpy observed for picric acid. In contrast, the low values of the correlation coefficients calculated for $\mathrm{PH}$ data come as no surprise in view of the large experimental uncertainties. Specially poor correlation observed for ortho compounds is consistent with the assumption of previous authors that interactions between nitro groups and ortho substituents make decomposition enthalpies harder to rationalize [13].

Insight into the predictive value of present equations is provided by the correlation between $\Delta_{d} H$ (DSC) and $(\chi / \eta) \Delta_{d} H^{0}$ illustrated in Figure 3. Again, it is interesting to distinguish experimental values according to the corresponding sample cells. Furthermore, after discarding $\mathrm{PH}$ data for ortho compounds, as done previously [13], a striking correlation $R^{2}=0.86$ is observed for the 64 remaining enthalpies measured in either kind of sample cells. This achievement is all the more remarkable as a single descriptor is used. For comparison, the corresponding determination coefficient between these 64 values and $\Delta_{d} H^{0}$ is $R^{2}=0.80$.

5.3. Predicting DSC Decomposition Enthalpies. These coefficients reflect the predictive value of present approaches. In order to carry out a rigorous comparison with previous QSPR methods, (2) and (4) have been fitted and validated against previously introduced training and test sets. However, 


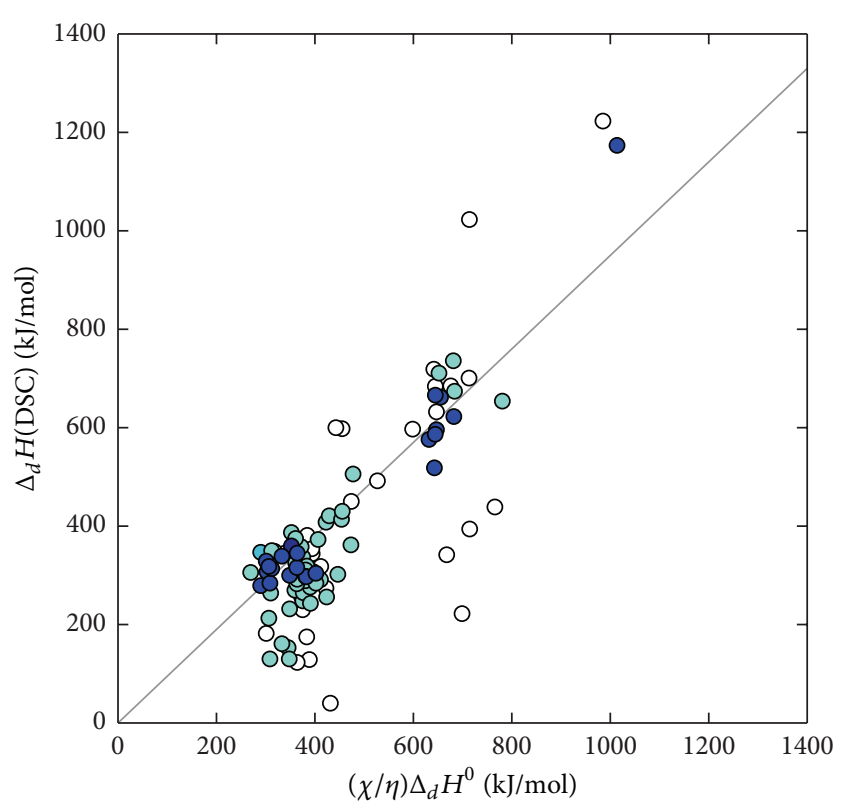

O $\mathrm{PH}$ (ortho)
O $\mathrm{PH}$ (nonortho)
- SC

FIGURE 3: Observed DSC decomposition enthalpies as a function of the descriptor employed in (4).

because present equations involve only a single adjustable parameter, they can be fitted against a much smaller training set, thus allowing for a more extensive validation of their predictive value. For this purpose, we have fitted both models against a training set of only 5 compounds and used the 94 remaining compounds as an external test set. This partition and corresponding results are detailed in the appendix.

The relative performances of the various methods are summarized in Table 1. $R^{2}$ stands for the determination coefficient between fitted and observed data. Although they are mostly irrelevant for present approaches based on a single linear parameter, corresponding coefficients derived from leave-one-out cross validations $\left(Q^{2}\right)$ and application of the models to external test sets $\left(R_{x}^{2}\right)$ are reported as well for the sake of comparison with QSPR methods. These coefficients should only be used to assess the relative performance of various models though a comparison of values obtained for the same data set. In fact, their individual values are deprived of significance in view of the fact that the distribution of $\Delta_{d} H$ (DSC) values in any set considered is far from Gaussian. Therefore, average absolute errors of the models are also reported in Table 1 for both the training set $\left(\mathrm{AAE}_{\mathrm{tr}}\right)$ and the test (validation) set $\left(\mathrm{AAE}_{v}\right)$. The number of variables of each model $(n)$ and the number of data included, respectively, in the training $\left(N_{\text {tr }}\right)$ and test $\left(N_{v}\right)$ sets are also provided in this table.

In principle, the predictive value of the models is best characterized by $R_{x}^{2}$ and $\mathrm{AAE}_{v}$. However, this is not necessarily the case in practice owing to the small size of most test sets considered, with only 8-11 data values for the most specialized models [13]. This is reflected by the fact that $R_{x}^{2}$ and $\mathrm{AAE}_{v}$ values sometimes provide more optimistic expectations than statistical parameters derived from the training set. In such cases, the least optimistic criteria are clearly to be considered.

Because SC data appear easier to rationalize, present equations are first fitted against the SC data set considered in previous papers $[8-10,12]$. The small size of this set (22 enthalpies) does not allow a rigorous assessment of the predictive value of QSPR methods. However, according to $Q^{2}$ coefficients, (4) taking reactivity into account appears better than (2). The only QSPR models with $Q^{2}$ values higher than the present ones were obtained in [10] as the outcome of an extensive screening of descriptors combinations. However, (2) and (4) provide average relative errors of, respectively, 13\% and $10 \%$ on this data set. Keeping in mind that such deviations are comparable to experimental uncertainties, the very high determination coefficients reported in [10] probably reflect overfitting issues. Furthermore, the corresponding models proved deprived of predictive value for present $\mathrm{PH}$ data, for which a value of $R_{x}^{2}$ as low as 0.24 was reported [13].

For $\mathrm{PH}$ data, present equations are first fitted independently for nonortho and ortho compounds, as done in [13]. For nonortho compounds, the best correlation between measured and predicted values $\left(R_{x}^{2}=0.92\right)$ is obtained using (4). According to the data reported in Table 1 , especially average errors $\mathrm{AAE}_{\text {tr }}$ and $\mathrm{AAE}_{v}$ for the training and test sets, this equation and the QSPR method of [13] exhibit similar performances. In contrast, both approaches fail to provide valuable predictions for ortho compounds. As a result, they perform poorly when applied to the prediction of $\mathrm{PH}$ data for arbitrary compounds.

In order to better characterize the value of present equations, they are eventually fitted against a small training set of five SC values defined in Table 2. The performances of the two resulting models are summarized in the last six rows of Table 1. Despite poorer fits against the 5 compounds in the training set, (4) yields systematically better predictions than (2), which suggests that reactivity indexes are indeed useful to obtain improved predictions. This is confirmed by the fits of the two equations against the whole set of 22 SC data. Finally, it may be noted that the reliability of both models steadily decreases ongoing from SC data to $\mathrm{PH}$ data for nonortho compounds to $\mathrm{PH}$ data for ortho compounds.

Present results demonstrate that the present approach makes it straightforward to derive predictive tools performing similarly or even better than the best QSPR methods at hand, provided $\mathrm{PH}$ data for ortho compounds are excluded from the data set. Whenever these difficult cases are considered, present equations fail to provide meaningful predictions, while the most successful QSPR studies appear to succeed in obtaining fairly reasonable results, especially for the seven-parameters model put forward very recently in [15].

These findings are understandable. Differences between SC and PH data can only be explained by complex processes, including heat and mass transfers within the cell, energy losses through sublimation, and evaporation, which are deliberately ignored by the present approach. In view 
TABLE 1: Comparison of presently available predictive methods. The second column indicates which kind of measurements (SC and/or PH) are used to assess the predictive value of the method. In the third column, $y / n$ indicates whether (y) or not (n) PH data for ortho compounds are included in the test set. In the last column, $c$ stands for the empirical parameter of present models; that is, it is equal to either $f$ when (2) is used or $a(\chi / \eta)$ when using (4). $\mathrm{AAE}_{\mathrm{tr}}$ and $\mathrm{AAE}_{v}$ values are in $\mathrm{kJ} / \mathrm{mol}$.

\begin{tabular}{|c|c|c|c|c|c|c|c|c|c|c|c|}
\hline Model reference & Cell & $\mathrm{PH} /$ ortho & $N_{\text {tr }}$ & $N_{v}$ & $n$ & $R^{2}$ & $Q^{2}$ & $R_{x}^{2}$ & $\mathrm{AAE}_{\mathrm{tr}}$ & $\mathrm{AAE}_{v}$ & $c$ \\
\hline \multicolumn{12}{|c|}{ Models for SC data } \\
\hline Reference [9] & SC & $\mathrm{n}$ & 22 & - & 6 & 0.91 & 0.84 & - & - & - & \\
\hline Reference [8] & & & & - & 3 & 0.85 & 0.73 & - & 65 & - & \\
\hline Reference [10] & & & & - & 3 & 0.98 & 0.97 & - & 22 & - & \\
\hline Reference $[10]$ & & & & - & 4 & 0.98 & 0.97 & - & 20 & - & \\
\hline Reference [12] & SC & $\mathrm{n}$ & 22 & - & 3 & 0.93 & 0.86 & - & - & - & \\
\hline Equation (2) & & & & - & 1 & 0.90 & 0.86 & - & 57 & - & 0.64 \\
\hline Equation (4) & & & & - & 1 & 0.93 & 0.91 & - & 43 & - & 0.97 \\
\hline \multicolumn{12}{|c|}{ Models for PH data and nonortho compounds } \\
\hline Reference [13] & $\mathrm{PH}$ & $\mathrm{n}$ & 31 & 11 & 4 & 0.91 & 0.86 & 0.84 & 68 & 56 & \\
\hline Equation (2) & & & & & 1 & 0.67 & 0.63 & 0.90 & 72 & 75 & 0.54 \\
\hline Equation (4) & & & & & 1 & 0.69 & 0.66 & 0.92 & 65 & 65 & 0.85 \\
\hline \multicolumn{12}{|c|}{ Models for PH data and ortho compounds } \\
\hline Reference [13] & $\mathrm{PH}$ & $\mathrm{y}$ & 27 & 8 & 4 & 0.94 & 0.91 & 0.42 & 55 & 112 & \\
\hline Equation (2) & & & & & 1 & 0.68 & 0.61 & 0.30 & 140 & 130 & 0.61 \\
\hline Equation (4) & & & & & 1 & 0.66 & 0.60 & 0.24 & 126 & 138 & 0.93 \\
\hline \multicolumn{12}{|c|}{ Generic models for $\mathrm{PH}$ data } \\
\hline Reference [13] & $\mathrm{PH}$ & $\mathrm{y}$ & 58 & 19 & 4 & 0.84 & 0.81 & 0.43 & 65 & 96 & \\
\hline Equation (2) & & & & & 1 & 0.69 & 0.65 & 0.44 & 105 & 100 & 0.58 \\
\hline Equation (4) & & & & & 1 & 0.68 & 0.65 & 0.41 & 95 & 94 & 0.89 \\
\hline Reference [14] & $\mathrm{PH}$ & $\mathrm{y}$ & 55 & 22 & 7 & 0.77 & 0.64 & 0.70 & 66 & 92 & \\
\hline Reference [14] & & & & & 4 & 0.76 & 0.66 & 0.67 & 66 & - & \\
\hline Reference [14] & & & & & 7 & 0.74 & $0.68^{\mathrm{a}}$ & 0.75 & 67 & 86 & \\
\hline Reference [15] & & & & & 7 & 0.86 & 0.79 & 0.84 & 53 & 82 & \\
\hline Reference [15] & & & & & 4 & 0.76 & 0.70 & 0.79 & 65 & 88 & \\
\hline Equation (2) & & & & & 1 & 0.59 & 0.55 & 0.70 & 92 & 129 & 0.54 \\
\hline Equation (4) & & & & & 1 & 0.58 & 0.54 & 0.68 & 88 & 114 & 0.84 \\
\hline \multicolumn{12}{|c|}{ Generic models for all compounds/sample cells, fitted using SC data } \\
\hline Equation (2) & SC & $\mathrm{n}$ & 5 & 17 & 1 & 0.94 & 0.88 & 0.90 & 40 & 54 & 0.61 \\
\hline Equation (4) & & & & & 1 & 0.91 & 0.85 & 0.94 & 40 & 43 & 0.95 \\
\hline Equation (2) & $\mathrm{SC}+\mathrm{PH}$ & $\mathrm{n}$ & 5 & 59 & 1 & 0.94 & 0.88 & 0.81 & 40 & 74 & 0.61 \\
\hline Equation (4) & & & & & 1 & 0.91 & 0.85 & 0.84 & 40 & 64 & 0.95 \\
\hline Equation (2) & $\mathrm{SC}+\mathrm{PH}$ & $\mathrm{y}$ & 5 & 94 & 1 & 0.94 & 0.88 & 0.68 & 40 & 98 & 0.61 \\
\hline Equation (4) & & & & & 1 & 0.91 & 0.85 & 0.68 & 40 & 88 & 0.95 \\
\hline
\end{tabular}

${ }^{\mathrm{a}}$ 7-fold cross validation.

of the correlation reported in Figure 1, such effects mostly affect PH data, especially for ortho compounds. Because reactivity, as described here with the help of $\chi$ and $\eta$, is only one factor among many others that may contribute to the difference between $\Delta_{d} H$ (DSC) and $\Delta_{d} H^{0}$, it is not surprising that present models perform better for SC data.
On the other hand, in view of the ability of QSPR techniques to learn from the data at hand and to account implicitly for complex and poorly known processes through an automatic selection of suitable descriptors, it comes as no surprise that they are especially competitive with the present semiempirical approach in the most difficult cases of $\mathrm{PH}$ data for ortho compounds. 


\section{Discussion}

The present work opens new perspectives with regard to $\Delta_{d} H$ (DSC) prediction in the context of thermal hazards assessment. Indeed, it can be noted that, at least for SC data, present descriptors $\Delta_{d} H^{0}$ and possibly $(\chi / \eta) \Delta_{d} H^{0}$ exhibit striking correlations with experiment. With determination coefficients of, respectively, 0.90 and 0.93 , these two descriptors correlate better with measured $\Delta_{d} H$ (DSC) data than any individual descriptor previously considered for QSPR analyses of this particular data set $[8,9]$. This suggests that future QSPR approaches should include them in the pool of descriptors. Alternatively, it is tempting to resort to the QSPR machinery to estimate the ratio (or the difference) between $\Delta_{d} H^{0}$ and $\Delta_{d} H$ (DSC), hence focusing the power of such techniques on the most challenging aspects of DSC decomposition enthalpies.

The present semiempirical approach is therefore complementary to mainstream QSPR techniques. It provides general equations involving complex quantities that should lend themselves to QSPR prediction. The originality of present models with respect to previous ones appears clearly in Table 1. Because they involve a single adjustable parameter, they can be fitted against small training sets and assessed using extended test sets. They are extremely robust and provide reliable predictions whenever a good fit of the training set is obtained. However, they cannot capture the complex processes that may affect decomposition enthalpies derived from DSC experiments.

Notwithstanding the originality of present approaches due to their less empirical character compared to previous ones, another distinctive feature of the present work is the fact that SC and PH data are considered simultaneously. Initially, the development of distinct models for these two kinds of measurements was motivated by the fairly large differences observed between SC and PH data obtained for the same compound, as those mentioned in the introduction. However, one may wonder whether such differences, up to about $220 \mathrm{~kJ} / \mathrm{mol}$, are actually significant. For practical reasons, it may be necessary in some cases to use either SC or $\mathrm{PH}$ sample cell, depending on the behavior of the compound under study. For instance, SC cells may be required for highly volatile compounds [4]. As a consequence, it is not possible to define a standard sample cell that would be used for all thermal characterization studies. In the context of hazard evaluation, we must therefore be satisfied with comparison involving $\mathrm{SC}$ as well as $\mathrm{PH}$ data.

Among present compounds, 15 have been experimentally characterized using both SC and PH sample cells. Figure 4 compares the two kinds of measurements. They clearly exhibit a significant correlation $\left(R^{2}=0.73\right)$ because the two dinitro compounds considered exhibit significantly higher decomposition enthalpies than mononitro compounds, regardless of the sample cell used. However, focusing on mononitro compounds, the correlation between both sets of measurements happens to be negative. Whereas the decomposition enthalpy of 4-nitroaniline is $224 \mathrm{~kJ} / \mathrm{mol}$ larger than for 2-nitrophenol according to $\mathrm{PH}$ data, it is $66 \mathrm{~kJ} / \mathrm{mol}$ lower according to SC data. Therefore, it is difficult to conclude regarding the relative severity of thermal hazards associated with these two compounds. In other words, enthalpy differences $<250 \mathrm{~kJ} / \mathrm{mol}$ appear to be insignificant.

In fact, even larger differences between $\mathrm{SC}$ and $\mathrm{PH}$ data were observed by Ando et al. [4]. They are explained by these authors in terms of evaporation of volatile species though pin-hole or the presence of residual air in sealed sample cells that might initiate an oxidation. In view of such results, $\Delta_{d} H^{0}$ might prove a valuable alternative to DSC data in view of characterizing the severity of hazards associated with runaway reactions.

\section{Conclusions}

This work confirms that $\Delta_{d} H^{0}$ is a major contribution to $\Delta_{d} H$ (DSC) and should be explicitly taken into account in order to estimate this experimental property. It points to a number of approaches that should yield improved results compared to current applications of the QSPR methodology to $\Delta_{d} H$ (DSC), including the development of semiempirical models along present lines or the application of QSPR to quantities related to the difference between $\Delta_{d} H^{0}$ and $\Delta_{d} H$ (DSC).

On the other hand, the present work establishes that decomposition enthalpies measured using pin-hole sample cells are especially difficult to rationalize as they represent widely varying fractions of $\Delta_{d} H^{0}$. This result provides a clear justification for the opposition of some researchers, mentioned in [4], to the use of pin-hole cells. In view of the fact that $\Delta_{d} H^{0}$ correlates better with data measured in sealed cells than values measured in pin-hole cells, present results suggest that $\Delta_{d} H^{0}$ provide an equally reliable thermal hazard indicator compared to DSC enthalpies. In practice, this does not question the usefulness of DSC analyses which remain necessary to determine decomposition temperatures and monitor the thermal events that may occur to a given material under heating.

Finally, the fact that a small but systematic improvement is obtained on introducing reactivity indices supports the underlying assumption that species whose decomposition occurs at relatively low temperatures tend to release a more significant fraction of their energy. In addition, the analysis of the measurements carried out in pin-hole cells supports the view that they are especially difficult to rationalize for compounds with substituents in ortho positions with respect to nitro groups, presumably as a result of reactions between these neighboring substituents.

\section{Appendix}

\section{Detailed Outcome of Present Models}

See Tables 2, 3, and 4 .

\section{Conflict of Interests}

The author declares that there is no conflict of interests regarding the publication of this paper. 


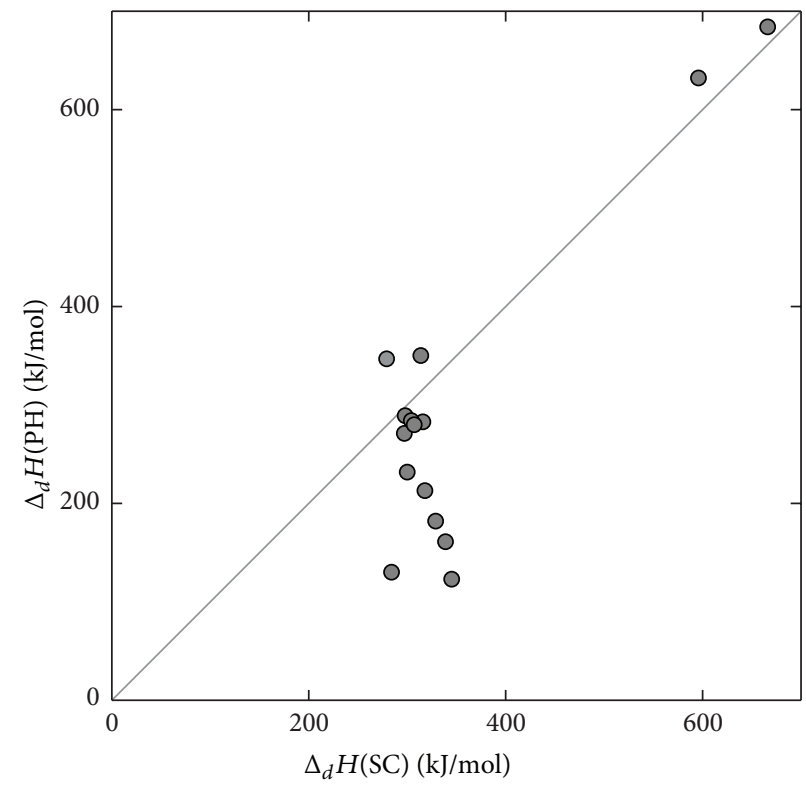

FIGURE 4: DSC decomposition enthalpies: PH versus SC values.

TABLE 2: Properties of the 22 compounds studied in sealed sample cells: name, electronegativity $\chi$, chemical hardness $\eta$, calculated solid-state formation enthalpy $\Delta_{f} H^{0}$, maximal energy content $\Delta_{d} H^{0}$, and DSC decomposition enthalpies $\Delta_{d} H(\mathrm{DSC})$ for the set of data measured in sealed sample cells [3]. All enthalpies are in $\mathrm{kJ} / \mathrm{mol}$. The stars indicate the five compounds included in the training set.

\begin{tabular}{|c|c|c|c|c|c|c|c|}
\hline Name & $\chi(\mathrm{eV})$ & $\eta(\mathrm{eV})$ & $\Delta_{f} H^{0}$ & $\Delta_{d} H^{0}$ & Equation (2) & Equation (4) & $\Delta_{d} H^{0}(\mathrm{DSC})[3]$ \\
\hline 4-Nitroaniline $(*)$ & 4.753 & 8.512 & 35 & 519 & 317 & 276 & 279 \\
\hline 1-Methyl-3-nitrobenzene & 5.417 & 9.152 & 38 & 521 & 318 & 294 & 284 \\
\hline 2-Nitrobenzoic acid & 5.917 & 9.500 & -286 & 614 & 375 & 364 & 297 \\
\hline 3-Nitrobenzoic acid & 5.983 & 9.490 & -296 & 604 & 369 & 363 & 298 \\
\hline 4-Nitrophenol & 5.372 & 9.003 & -119 & 584 & 357 & 332 & 300 \\
\hline$p$-Nitrobenzoic acid $(*)$ & 6.067 & 9.140 & -294 & 606 & 370 & 383 & 304 \\
\hline 2-Nitroaniline & 4.773 & 8.283 & 41 & 525 & 320 & 288 & 307 \\
\hline 3-Nitrobenzenamine & 4.763 & 8.129 & 49 & 533 & 325 & 297 & 314 \\
\hline 3-Nitrophenol & 5.378 & 8.758 & -111 & 592 & 361 & 346 & 316 \\
\hline 1-Methyl-4-nitrobenzene & 5.465 & 9.262 & 36 & 519 & 317 & 292 & 318 \\
\hline 1-Methyl-2-nitrobenzene $(*)$ & 5.319 & 9.296 & 42 & 526 & 321 & 287 & 329 \\
\hline Nitrobenzene & 5.603 & 9.434 & 77 & 561 & 342 & 317 & 339 \\
\hline 2-Nitrophenol & 5.372 & 8.602 & -120 & 583 & 356 & 347 & 345 \\
\hline 1-Chloro-4-nitrobenzene & 5.785 & 9.099 & 46 & 554 & 338 & 335 & 360 \\
\hline 1,2-Dinitrobenzene & 6.312 & 9.512 & 91 & 969 & 591 & 612 & 518 \\
\hline 2,6-Dinitrotoluene $(*)$ & 6.065 & 9.306 & 47 & 969 & 592 & 601 & 576 \\
\hline 1,3-Dinitrobenzene & 6.419 & 9.474 & 74 & 951 & 581 & 613 & 587 \\
\hline 2,4-Dinitrotoluene & 6.195 & 9.226 & 41 & 963 & 588 & 616 & 596 \\
\hline 1,4-Dinitrobenzene & 6.514 & 9.097 & 75 & 953 & 581 & 649 & 623 \\
\hline 2,4-Dinitrophenol & 6.096 & 8.796 & -128 & 945 & 577 & 624 & 662 \\
\hline 4-Methyl-1,2-dinitrobenzene $(*)$ & 6.113 & 9.225 & 50 & 973 & 594 & 614 & 666 \\
\hline Picric acid & 6.711 & 8.824 & -112 & 1333 & 814 & 965 & 1174 \\
\hline
\end{tabular}


TABLE 3: The same data as in Table 2 for the 42 nonortho compounds studied in pin-hole sample cells.

\begin{tabular}{|c|c|c|c|c|c|c|c|}
\hline Name & $\chi(\mathrm{eV})$ & $\eta(\mathrm{eV})$ & $\Delta_{f} H^{0}$ & $\Delta_{d} H^{0}$ & Equation (2) & Equation (4) & $\Delta_{d} H^{0}(\mathrm{DSC})[4]$ \\
\hline 1-Methyl-3-nitrobenzene & 5.417 & 9.152 & 38 & 521 & 318 & 294 & 130 \\
\hline 2-Amino-4-nitrophenol & 4.680 & 7.908 & -138 & 588 & 359 & 331 & 130 \\
\hline 2-Amino-5-nitrophenol & 4.820 & 8.121 & -143 & 582 & 355 & 329 & 153 \\
\hline Nitrobenzene & 5.603 & 9.434 & 77 & 561 & 342 & 317 & 161 \\
\hline 1-Methyl-4-nitrobenzene & 5.465 & 9.262 & 36 & 519 & 317 & 292 & 213 \\
\hline 4-Nitrophenol & 5.372 & 9.003 & -119 & 584 & 357 & 332 & 232 \\
\hline 1-Methoxy-3-nitrobenzene & 5.270 & 8.692 & -81 & 645 & 394 & 372 & 243 \\
\hline 1-Methoxy-4-nitrobenzene & 5.261 & 8.939 & -88 & 638 & 389 & 357 & 248 \\
\hline 3-Nitrobenzoic acid methyl ester & 5.920 & 9.467 & -267 & 678 & 414 & 403 & 256 \\
\hline 2,6-Dichloro-4-nitrobenzenamine & 5.178 & 8.332 & -32 & 499 & 305 & 295 & 264 \\
\hline 4-Nitrobenzene acetic acid & 5.679 & 9.367 & -324 & 620 & 379 & 358 & 265 \\
\hline 1-Ethoxy-4-nitrobenzene & 5.213 & 8.913 & -111 & 614 & 375 & 342 & 270 \\
\hline 1-(3-Nitrophenyl)-ethanone & 5.873 & 9.462 & -96 & 629 & 384 & 372 & 276 \\
\hline 4-Nitrophenylhydrazine & 4.868 & 8.426 & 163 & 647 & 395 & 356 & 277 \\
\hline 3-Nitrophenol & 5.378 & 8.758 & -111 & 592 & 361 & 346 & 283 \\
\hline$p$-Nitrobenzoic acid & 6.067 & 9.140 & -294 & 606 & 370 & 383 & 284 \\
\hline 3-Nitrobenzoic acid & 5.983 & 9.490 & -296 & 604 & 369 & 363 & 289 \\
\hline 1-(4-Nitrophenyl)-ethanone & 5.954 & 9.159 & -93 & 633 & 386 & 392 & 291 \\
\hline 4-Nitrobenzyl alcohol & 5.649 & 9.319 & -125 & 600 & 367 & 346 & 292 \\
\hline 4-Nitrobenzoic acid methyl ester & 6.007 & 9.149 & -265 & 680 & 415 & 425 & 302 \\
\hline 2-Methyl-4-nitroaniline & 4.687 & 8.421 & 1 & 484 & 296 & 257 & 306 \\
\hline 3-Nitrobenzamide & 5.924 & 9.492 & -115 & 611 & 373 & 363 & 311 \\
\hline 4-Nitrobenzamide & 5.903 & 9.355 & -118 & 608 & 371 & 365 & 319 \\
\hline 3-Nitrobenzyl alcohol & 5.395 & 9.169 & -112 & 614 & 375 & 344 & 325 \\
\hline 1-Chloromethyl-4-nitrobenzene & 5.261 & 8.940 & -88 & 638 & 389 & 357 & 337 \\
\hline 4-Nitroaniline & 4.753 & 8.512 & 35 & 519 & 317 & 276 & 347 \\
\hline 3-Nitrobenzenamine & 4.763 & 8.129 & 49 & 533 & 325 & 297 & 350 \\
\hline 3-Nitrobenzene acetic acid & 5.604 & 9.352 & -324 & 621 & 379 & 354 & 358 \\
\hline 4-Nitrobenzoic acid hydrazide & 5.911 & 9.088 & 2 & 727 & 444 & 450 & 362 \\
\hline N-(3-Nitrophenyl)-Acetamide & 5.099 & 8.449 & -129 & 597 & 364 & 343 & 369 \\
\hline 3-Nitrobenzaldehyde & 5.915 & 9.467 & -52 & 650 & 397 & 387 & 373 \\
\hline 2-Methoxy-5-nitrobenzenamine & 4.597 & 7.898 & -106 & 620 & 378 & 343 & 375 \\
\hline N-(4-Nitrophenyl)-acetamide & 5.130 & 8.596 & -135 & 590 & 360 & 335 & 387 \\
\hline 4-Nitrobenzoyl chloride & 6.383 & 9.040 & -106 & 598 & 365 & 402 & 408 \\
\hline 3-(3-Nitrophenyl)-2-propenoic acid & 5.627 & 8.753 & -238 & 706 & 431 & 432 & 414 \\
\hline 4-Nitrobenzaldehyde & 5.998 & 9.143 & -49 & 654 & 399 & 408 & 421 \\
\hline 3-Nitrobenzhydrazide & 5.854 & 9.303 & -2 & 724 & 442 & 434 & 430 \\
\hline 3-(4-Nitrophenyl)-2-propenoic acid & 5.808 & 8.601 & -238 & 707 & 432 & 455 & 506 \\
\hline 3,5-Dinitrobenzonitrile & 6.777 & 9.375 & 225 & 1080 & 659 & 743 & 654 \\
\hline 3,5-Dinitrobenzoic acid & 6.707 & 9.605 & -292 & 979 & 597 & 651 & 674 \\
\hline 1-(Chloromethyl)-3,5-dinitrobenzene & 6.452 & 9.250 & 11 & 935 & 571 & 621 & 711 \\
\hline 3,5-Dinitrobenzamide & 6.561 & 9.429 & -118 & 979 & 598 & 648 & 736 \\
\hline
\end{tabular}


TABLE 4: Same data as in Table 2 for the 35 ortho compounds studied in pin-hole sample cells.

\begin{tabular}{|c|c|c|c|c|c|c|c|}
\hline Name & $\chi(\mathrm{eV})$ & $\eta(\mathrm{eV})$ & $\Delta_{f} H^{0}$ & $\Delta_{d} H^{0}$ & Equation (2) & Equation (4) & $\Delta_{d} H^{0}$ (DSC) $[4]$ \\
\hline 5-Chloro-2-nitrobenzotrifluoride & 6.208 & 9.080 & -606 & 631 & 385 & 411 & 40 \\
\hline 2-Nitrophenol & 5.372 & 8.602 & -120 & 583 & 356 & 347 & 123 \\
\hline 4-Fluoro-2-nitrotoluene & 5.547 & 8.856 & -136 & 621 & 379 & 370 & 129 \\
\hline 2-Nitrobenzene acetic acid & 5.618 & 9.265 & -312 & 632 & 386 & 365 & 175 \\
\hline 1-Methyl-2-nitrobenzene & 5.319 & 9.296 & 42 & 526 & 321 & 287 & 182 \\
\hline 2,6-Dinitrobenzoic acid & 6.657 & 9.532 & -270 & 1001 & 611 & 665 & 222 \\
\hline 2-Nitroanisole & 5.135 & 8.911 & -75 & 651 & 397 & 357 & 230 \\
\hline 2-Nitrobenzamide & 5.776 & 9.554 & -108 & 617 & 377 & 355 & 256 \\
\hline 2-nitrobenzoic acid & 5.917 & 9.500 & -286 & 614 & 375 & 364 & 271 \\
\hline 2-Nitrobenzoic acid, methyl ester & 5.841 & 9.510 & -257 & 688 & 420 & 402 & 274 \\
\hline 2-Nitroaniline & 4.773 & 8.283 & 41 & 525 & 320 & 288 & 280 \\
\hline $\mathrm{N}$-(2-Nitrophenyl)-acetamide & 5.133 & 8.292 & -128 & 597 & 365 & 352 & 297 \\
\hline 2-Nitroacetophenone & 5.798 & 9.399 & -85 & 641 & 391 & 376 & 308 \\
\hline 2-Nitrobenzaldehyde & 5.846 & 9.376 & -42 & 660 & 403 & 392 & 318 \\
\hline 2-Nitrobenzenemethanol & 5.646 & 9.284 & -113 & 613 & 374 & 355 & 319 \\
\hline 4-Chloro-1,2-dinitrobenzene & 6.367 & 9.024 & 68 & 946 & 578 & 636 & 342 \\
\hline 2-Nitrobenzenesulfenylchloride & 5.355 & 7.735 & 62 & 570 & 348 & 375 & 344 \\
\hline 3-Methyl-4-nitrophenol & 5.301 & 9.002 & -150 & 576 & 351 & 323 & 345 \\
\hline 4-Chloro-2-nitrobenzenamine & 4.972 & 8.098 & 10 & 517 & 316 & 302 & 349 \\
\hline 4-Chloro-3-nitrobenzoic acid & 6.012 & 9.049 & -308 & 593 & 362 & 375 & 354 \\
\hline (2-Nitrophenyl)-hydrazine & 4.722 & 8.126 & 178 & 661 & 404 & 366 & 381 \\
\hline 2,4-Dinitrobenzoic acid & 6.735 & 9.318 & -282 & 988 & 603 & 680 & 394 \\
\hline 1,5-Difluoro-2,4-dinitrobenzene & 6.747 & 9.290 & -279 & 1054 & 644 & 729 & 439 \\
\hline 4-Hydroxy-3-methoxy-5-nitrobenzaldehyde & 5.356 & 8.513 & -388 & 753 & 460 & 451 & 450 \\
\hline 2-Nitro-5-thiocyanatobenzoic acid & 5.702 & 8.277 & -112 & 765 & 467 & 502 & 492 \\
\hline 2,4-Dinitrobenzenamine & 5.501 & 8.493 & 24 & 924 & 564 & 570 & 597 \\
\hline 2-Nitrobenzoic acid, hydrazide & 5.647 & 9.111 & 9 & 734 & 448 & 433 & 598 \\
\hline 2-Nitrocinnamic acid & 5.634 & 9.059 & -233 & 712 & 434 & 421 & 600 \\
\hline 2,4-Dinitrotoluene & 6.195 & 9.226 & 41 & 963 & 588 & 616 & 632 \\
\hline 4-Methyl-1,2-dinitrobenzene & 6.113 & 9.225 & 50 & 973 & 594 & 614 & 684 \\
\hline 3,4-Dinitrobenzenemethanol & 6.100 & 9.199 & -100 & 1019 & 622 & 643 & 685 \\
\hline 3,4-Dinitrobenzoic acid & 6.684 & 9.341 & -274 & 996 & 608 & 679 & 701 \\
\hline 2,6-Dinitrobenzenamine & 5.580 & 8.103 & 32 & 932 & 569 & 611 & 719 \\
\hline 2-Chloro-3,5-dinitrobenzoic acid & 6.614 & 9.046 & -296 & 976 & 596 & 680 & 1023 \\
\hline 2-Methyl-1,3,5-trinitrobenzene & 6.807 & 9.328 & 57 & 1350 & 824 & 938 & 1223 \\
\hline
\end{tabular}

\section{Acknowledgment}

The author is grateful to his colleagues from INERIS, authors of $[8-10,13,14]$, for drawing his attention to the needs arising from the REACH regulatory framework.

\section{References}

[1] F. A. Quintero, S. J. Patel, F. Muñoz, and M. Sam Mannan, "Review of existing QSAR/QSPR models developed for properties used in hazardous chemicals classification system," Industrial and Engineering Chemistry Research, vol. 51, no. 49, pp. 16101-16115, 2012.
[2] D. A. Crowl and T. I. Elwell, "Identifying criteria to classify chemical mixtures as 'highly hazardous' due to chemical reactivity," Journal of Loss Prevention in the Process Industries, vol. 17, no. 4, pp. 279-289, 2004.

[3] Y.-S. Duh, C. Lee, C.-C. Hsu, D.-R. Hwang, and C.-S. Kao, "Chemical incompatibility of nitrocompounds," Journal of Hazardous Materials, vol. 53, no. 1-3, pp. 183-194, 1997.

[4] T. Ando, Y. Fujimoto, and S. Morisaki, "Analysis of differential scanning calorimetric data for reactive chemicals," Journal of Hazardous Materials, vol. 28, no. 3, pp. 251-280, 1991.

[5] D. J. Frurip and T. Elwell, "Effective use of differential scanning calorimetry in reactive chemicals hazard evaluation," Process Safety Progress, vol. 26, no. 1, pp. 51-58, 2007. 
[6] D. Mathieu, "Formation enthalpies derived from pairwise interactions: a step toward more transferable reactive potentials for organic compounds," Journal of Chemical Theory and Computation, vol. 8, no. 4, pp. 1295-1303, 2012.

[7] Y. Lu, D. Ng, and M. S. Mannan, "Prediction of the reactivity hazards for organic peroxides using the QSPR approach," Industrial and Engineering Chemistry Research, vol. 50, no. 3, pp. 1515-1522, 2011.

[8] G. Fayet, L. Joubert, P. Rotureau, and C. Adamo, "On the use of descriptors arising from the conceptual density functional theory for the prediction of chemicals explosibility," Chemical Physics Letters, vol. 467, no. 4-6, pp. 407-411, 2009.

[9] G. Fayet, P. Rotureau, L. Joubert, and C. Adamo, "On the prediction of thermal stability of nitroaromatic compounds using quantum chemical calculations," Journal of Hazardous Materials, vol. 171, no. 1-3, pp. 845-850, 2009.

[10] G. Fayet, P. Rotureau, L. Joubert, and C. Adamo, "QSPR modeling of thermal stability of nitroaromatic compounds: DFT vs. AM1 calculated descriptors," Journal of Molecular Modeling, vol. 16, no. 4, pp. 805-812, 2010.

[11] P. Sang, J.-W. Zou, X. Lin, and P. Zhou, "Linear and nonlinear QSPR models for predicting thermal stabilities of nitroaromatic compounds," Chemical Research in Chinese Universities, vol. 27, no. 5, pp. 891-895, 2011.

[12] P. Sang, J. W. Zou, L. Xu, and Y. H. Liu, "QSPR of thermal stability of nitroaromatic explosives using theoretical descriptors derived from electrostatic potentials on the molecular surface," Chinese Journal of Structural Chemistry, vol. 30, pp. 533-537, 2011.

[13] G. Fayet, P. Rotureau, L. Joubert, and C. Adamo, "Development of a QSPR model for predicting thermal stabilities of nitroaromatic compounds taking into account their decomposition mechanisms," Journal of Molecular Modeling, vol. 17, no. 10, pp. 2443-2453, 2011.

[14] G. Fayet, A. Del Rio, P. Rotureau, L. Joubert, and C. Adamo, "Predicting the thermal stability of nitroaromatic compounds using chemoinformatic tools," Molecular Informatics, vol. 30, no. 6-7, pp. 623-634, 2011.

[15] J. Z. Li, H. X. Liu, X. Huo, and P. Gramatica, "Structure-activity relationship analysis of the thermal stabilities of nitroaromatic compounds following different decomposition mechanisms," Molecular Informatics, vol. 32, no. 2, pp. 193-202, 2013.

[16] J. Akhavan, The Chemistry of Explosives, The Royal Society of Chemistry, London, UK, 1998.

[17] E. F. C. Byrd and B. M. Rice, "Improved prediction of heats of formation of energetic materials using quantum mechanical calculations," Journal of Physical Chemistry A, vol. 110, no. 3, pp. 1005-1013, 2006.

[18] L. Glasser and H. D. B. Jenkins, "Volume-based thermodynamics: a prescription for its application and usage in approximation and prediction of thermodynamic data," Journal of Chemical and Engineering Data, vol. 56, no. 4, pp. 874-880, 2011.

[19] U. Preiss, S. Bulut, and I. Krossing, "In silico prediction of the melting points of ionic liquids from thermodynamic considerations: a case study on 67 salts with a melting point range of $337^{\circ} \mathrm{C}$," Journal of Physical Chemistry B, vol. 114, no. 34, pp. 11133-11140, 2010.

[20] U. P. Preiss, W. Beichel, A. M. T. Erle, Y. U. Paulechka, and I. Krossing, "Is universal, simple melting point prediction possible?” ChemPhysChem, vol. 12, no. 16, pp. 2959-2972, 2011.
[21] B. Lian and S. H. Yalkowsky, "Molecular geometry and melting point related properties," Industrial and Engineering Chemistry Research, vol. 51, no. 51, pp. 16750-16754, 2012.

[22] D. Mathieu, "Prediction of gurney parameters based on an analytic description of the expanding products," Journal of Energetic Materials, vol. 33, no. 2, pp. 102-115, 2014.

[23] D. Mathieu, "Inductive modeling of physico-chemical properties: flash point of alkanes," Journal of Hazardous Materials, vol. 179, no. 1, pp. 1161-1164, 2010.

[24] D. Mathieu, "Power law expressions for predicting lower and upper flammability limit temperatures," Industrial and Engineering Chemistry Research, vol. 52, no. 26, pp. 9317-9322, 2013.

[25] D. Mathieu and T. Alaime, "Predicting impact sensitivities of nitro compounds on the basis of a semi-empirical rate constant," The Journal of Physical Chemistry A, vol. 118, no. 41, pp. 97209726, 2014.

[26] F. Jensen, Introduction to Computational Chemistry, John Wiley \& Sons, New York, NY, USA, 1999.

[27] S. Zeman, "Modified Evans-Polanyi-Semenov relationship in the study of chemical micromechanism governing detonation initiation of individual energetic materials," Thermochimica Acta, vol. 384, no. 1-2, pp. 137-154, 2002.

[28] E. Theerlynck, D. Mathieu, and P. Simonetti, "Towards improved models to rationalize and estimate the decomposition temperatures of nitroalkanes, nitramines and nitric esters," Thermochimica Acta, vol. 426, no. 1-2, pp. 123-129, 2005.

[29] P. Geerlings, F. De Proft, and W. Langenaeker, "Conceptual density functional theory," Chemical Reviews, vol. 103, no. 5, pp. 1793-1873, 2003.

[30] M. J. Kamlet and S. J. Jacobs, "Chemistry of detonations. I. A simple method for calculating detonation properties of $\mathrm{C}-\mathrm{H}$ N-O explosives," Journal of Chemical Physics, vol. 48, no. 1, pp. 23-27, 1968.

[31] P. J. Linstrom and W. G. Mallard, Eds., NIST Chemistry WebBook, NIST Standard Reference Database no. 69, National Institute of Standard and Technology, Gaithersburg, Md, USA, 2011, http://webbook.nist.gov/.

[32] G. B. Rocha, R. O. Freire, A. M. Simas, and J. J. P. Stewart, "RM1: a reparameterization of $\mathrm{AM} 1$ for $\mathrm{H}, \mathrm{C}, \mathrm{N}, \mathrm{O}, \mathrm{P}, \mathrm{S}, \mathrm{F}, \mathrm{Cl}, \mathrm{Br}$, and I," Journal of Computational Chemistry, vol. 27, no. 10, pp. 1101-1111, 2006.

[33] J. J. P. Stewart, MOPAC7.0: A General Molecular Orbital Package, vol. 45, QCPE, 1993.

[34] D. Mathieu and Y. Pipeau, "Formation enthalpies of ions: routine prediction using atom equivalents," Journal of Chemical Theory and Computation, vol. 6, no. 7, pp. 2126-2139, 2010.

[35] D. Mathieu and P. Simonetti, "Evaluation of solid-state formation enthalpies for energetic materials and related compounds," Thermochimica Acta, vol. 384, no. 1-2, pp. 369-375, 2002.

[36] D. Mathieu, "Simple alternative to neural networks for predicting sublimation enthalpies from fragment contributions," Industrial and Engineering Chemistry Research, vol. 51, no. 6, pp. 2814-2819, 2012. 

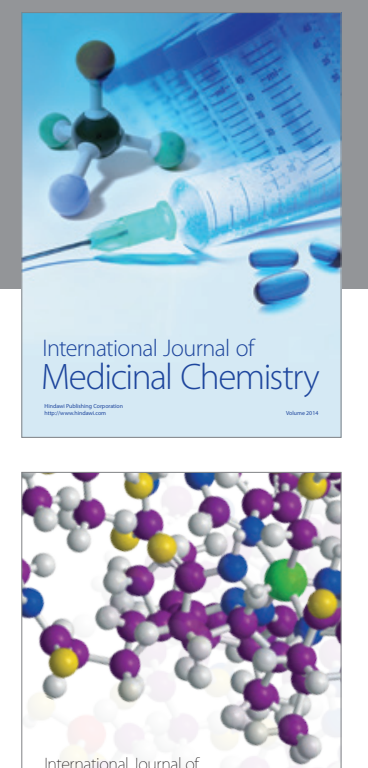

\section{Carbohydrate} Chemistry

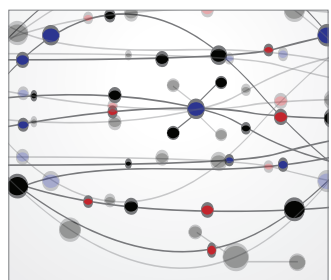

The Scientific World Journal
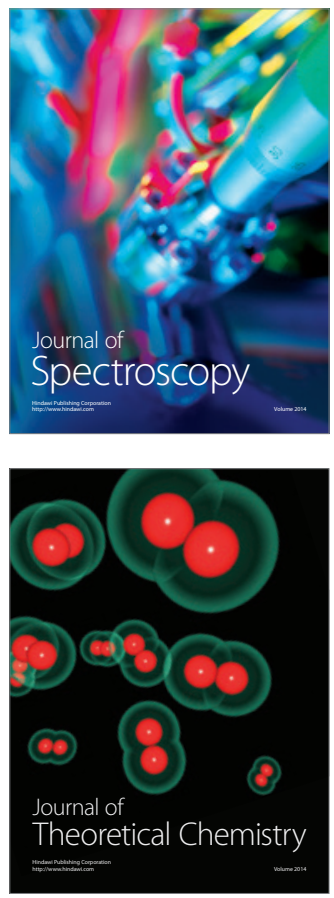
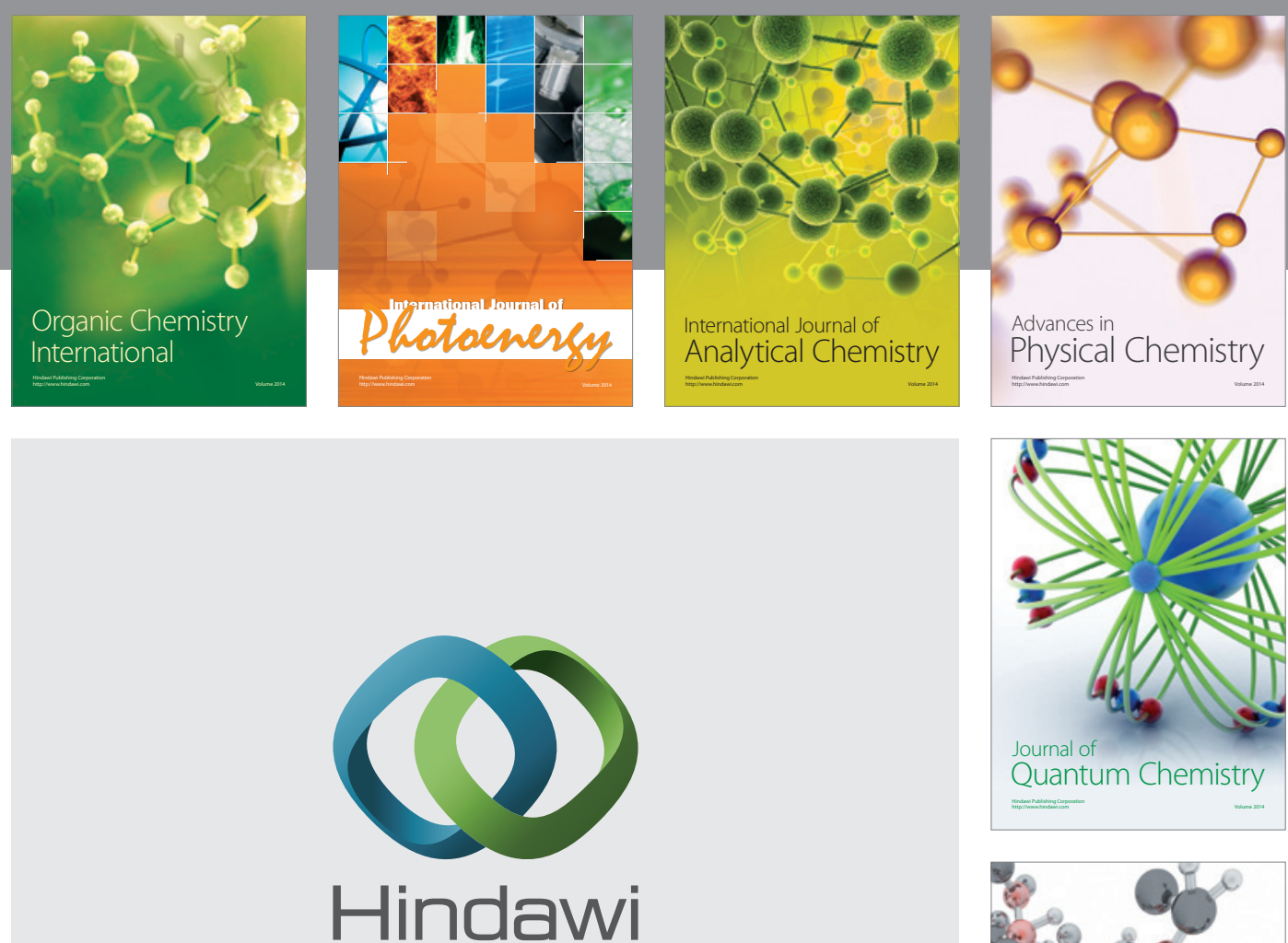

Submit your manuscripts at

http://www.hindawi.com

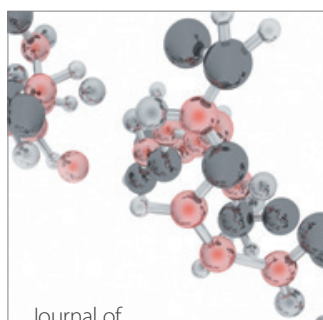

Analytical Methods

in Chemistry

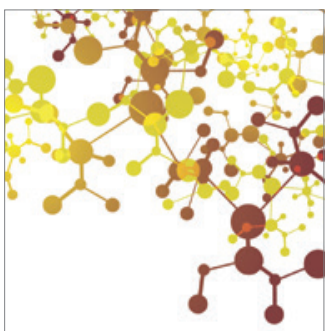

Journal of

Applied Chemistry

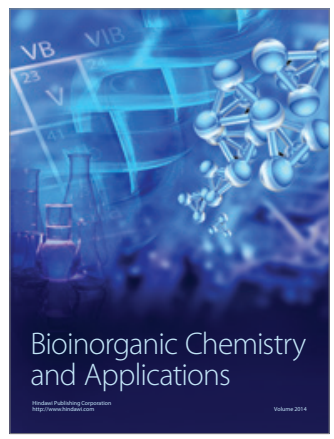

Inorganic Chemistry
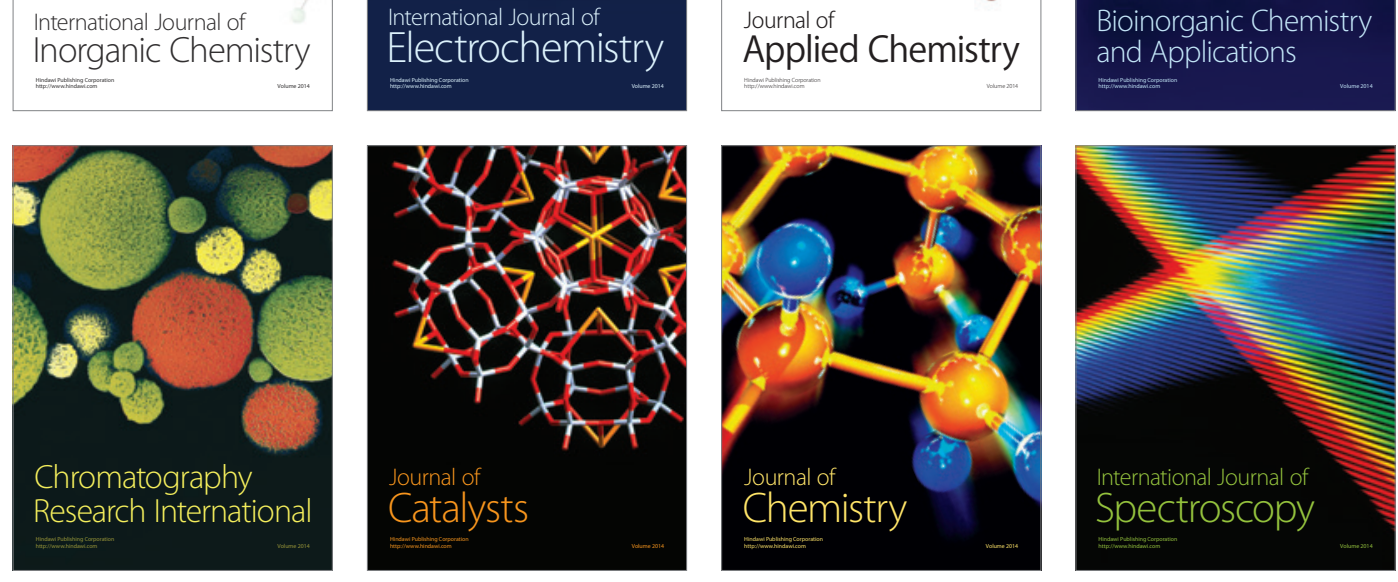